\title{
ON THE SPECTRAL DISSIPATION OF OCEAN WAVES DUE TO WHITE CAPPING*
}

\author{
KLAUS HASSELMANN \\ Universität Hamburg, Institut für Geophysik, F.R.G.
}

(Received 29 June, 1973)

\begin{abstract}
Summary. The effect of white capping on the spectral energy balance of surface waves is investigated by expressing the white-cap interactions in terms of an equivalent ensemble of random pressure pulses. It is shown first that the source function for any non-expansible interaction process which is weak-inthe-mean is quasi-linear. In the case of white capping, the damping coefficient is then shown to be proportional to the square of the frequency, provided the wave scales are large compared with the white-cap dimensions. The remaining free factor is determined indirectly from consideration of the spectral energy balance. The proposed white-capping dissipation function is consistent with the structure of the energy balance derived from JONSWAP, and the existence of a $\sigma^{-5}$ spectrum governed by a non-local energy balance between the atmospheric input, the nonlinear energy transfer and dissipation. However, closure of the energy balance involves hypotheses regarding the structure of the atmospheric input function which need to be tested by further measurements. The proposed set of source functions may nevertheless be useful for numerical wave-prediction. According to the model, nearly all the momentum transferred across the air-sea interface enters the wave field. For fetchlimited and fully developed spectra in a stationary, uniform wind field, the drag coefficient remains approximately constant. However, for more general wind conditions, this will not be the case and the wave spectrum should be included in an accurate parameterisation of the air-sea momentum transfer.
\end{abstract}

\section{Introduction}

Our understanding of the energy balance of surface-wave spectra has advanced considerably in recent years as a combined result of fundamental theoretical studies and detailed field investigations. With increasing insight into the structure of the source function governing the spectral energy balance, many of the empirical terms in numerical wave-prediction models have gradually been replaced by more reliable expressions derived from an analysis of the basic physical processes (Gelci et al., 1957; Hasselmann, 1960; Pierson et al., 1966; Darbyshire and Simpson, 1967; Inoue, 1967; Barnett, 1968; Gelci and Devillaz, 1970; Ewing, 1971). However, a persistent difficulty obstructing this development has been an appropriate representation of the source term representing the energy loss due to white capping. It is generally believed that white capping is the dominant dissipative mechanism in a wave field at moderate and higher wind speeds - simply because other dissipative processes such as molecular viscosity or turbulence (Phillips, 1959; Hasselmann, 1968) appear to be inadequate to remove the energy which is known to be imparted to the waves by the wind. Unfortunately, however, white capping represents a localized, strongly nonlinear interaction which cannot be treated by the standard perturbation techniques available for weak interactions and has therefore resisted a rigorous treatment.

* Contribution from the Sonderforschungsbereich 'Meeresforschung Hamburg' of the Deutsche Forschungsgemeinschaft. 
Two aspects of the problem may be distinguished. Firstly, the fluid mechanical process of white capping itself needs to be understood. Secondly, if the theory is to have application in wave prediction, the effect of a random ensemble of white caps on the energy balance of the wave spectrum must be expressed in terms of the wave statistics.

Most work on white capping has been concerned with the first question, in particular with the conditions for the onset of white capping and the characteristics of the limiting wave form (cf. Schwarz, 1972; Longuet-Higgins, 1973a, and references quoted in these papers). A first analysis of some of the flow properties of a spilling breaker has recently been undertaken by Longuet-Higgins (1973b).

Investigations of white capping in relation to the wave spectrum have concentrated hitherto on the concept of a high-frequency equilibrium range, rather than on the source function. Assuming that the equilibrium region is controlled entirely by wave breaking and that the process is local in wavenumber space, Phillips (1958) suggested that on dimensional grounds the one-dimensional frequency spectrum $F(\sigma)$ should be of the general form $F(\sigma)=\alpha g^{2} \sigma^{-5}$, where $g=$ gravitational acceleration, $\sigma=$ frequency and $\alpha$ is a universal constant. A theoretical estimate of $\alpha$ has been given by Longuet-Higgins (1969) on the basis of a specific model of the energy losses in a white cap, assuming a prescribed $\sigma^{-5}$ spectral distribution. However, recent extensive measurements of wave growth during the Joint North Sea Wave Project (JONSWAP, Hasselmann et al., 1973) indicate that the equilibrium range of the spectrum is not governed primarily by wave breaking, but represents a balance between several processes, including the non-local energy transfer from lower to higher wavenumbers by resonant third-order wave-wave interactions. The fetch dependence of $\alpha$ noted, for example, by Mitsuyasu (1968), (1969) in JONSWAP and by other workers also suggests that the $\sigma^{-5}$ power law cannot be explained by straight-forward dimensional arguments.

In this paper the effect of white capping on the spectral energy distribution is investigated in terms of the source function. The wave spectrum itself then follows from the energy-balance equation as the net response to a number of source functions, of which the white-capping term represents only one contribution. It is found that for the particular form derived for the white-capping source function, the white-capping losses are able to balance the energy and momentum input from the atmosphere plus the nonlinear transfer to shorter waves only for a particular shape of the equilibrium spectrum - which turns out to be approximately the $\sigma^{-5}$ distribution.

The structure of the white-capping source function is derived without entering into the details of the white-capping process itself. First it is shown that under very general conditions, the source function $S_{p}$ for any interaction process $p$ which is strongly nonlinear locally but still weak-in-the-mean is quasi-linear with respect to the wave spectrum, i.e., $S_{p}(\mathbf{k})=-\gamma E(\mathbf{k})$, where $\gamma$ is a functional of the entire wave spectrum. Assuming that the space-time scales of a white cap are small compared with the wavelengths and periods of the waves under consideration, the damping factor for white capping is then found to be of the form $\gamma=\eta \sigma^{2}$, where $\eta$ is a constant for a given wave 
state. Finally, $\eta$ is determined indirectly from consideration of the overall energy and momentum balance.

The form of the white-capping source function is in accordance with the picture of the energy and momentum balance of the wave spectrum derived from JONSWAP. A set of three source functions (wind input, nonlinear transfer and dissipation) is proposed which reproduces the JONSWAP results at intermediate and large fetches, including the transition to the fully developed Pierson-Moskowitz spectrum. The set provides a basis for numerical wave prediction, but is not free from hypotheses. This applies in particular to the form assumed for the input source function, which needs to be tested by independent field studies of the air-sea interaction processes.

\section{The Source Function for a Weak-in-the-Mean Interaction Process}

Consider a random, horizontally homogeneous wave field in an infinitely deep fluid. Assuming that to a first approximation the waves are linear and irrotational, the velocity potential $\varphi$, pressure $p$ and surface displacement $\zeta$ can be represented within a given area $-L / 2<x_{1}, x_{2}<L / 2$ as Fourier sums*

$$
\left\{\begin{array}{c}
\varphi \\
p \\
\zeta
\end{array}\right\}=\sum_{\mathbf{k}}\left\{\begin{array}{c}
\varphi_{\mathbf{k}}(t) e^{k z} \\
p_{\mathbf{k}}(t) e^{k z} \\
\zeta_{\mathbf{k}}(t)
\end{array}\right\} e^{i \mathbf{k} \cdot \mathbf{x}}
$$

where $\mathbf{x}=\left(x_{1}, x_{2}\right)$ denotes the horizontal coordinate vector and $z$ is the vertical coordinate, taken positive upwards.

If the waves are excited by a surface pressure field $\hat{p}=\sum \hat{p}_{\mathbf{k}}(t) e^{i \mathbf{k} \cdot \mathbf{x}}$, the equations of motion reduce to the well-known forced harmonic-oscillator equation (cf., Lamb, 1932)

$$
\frac{\partial^{2} \zeta_{\mathbf{k}}}{\partial t^{2}}+\sigma^{2} \zeta_{\mathbf{k}}=-k \hat{p}_{\mathbf{k}} / \varrho_{w},
$$

where $\sigma^{2}=g k$ and $\varrho_{w^{\prime}}=$ density of water.

After determining the surface elevation by integrating (2.1), the potential flow in the interior of the fluid follows from the Laplace equation with the appropriate linear kinematic boundary conditions at the surface and at $z=-\infty$,

$$
\begin{aligned}
& \nabla^{2} \varphi=0, \quad z<0 \\
& k \varphi_{\mathbf{k}}=\frac{\partial \zeta_{\mathbf{k}}}{\partial t}, \quad z=0 \\
& \varphi_{\mathbf{k}} \rightarrow 0, \quad z \rightarrow-\infty .
\end{aligned}
$$

* As usual, Fourier-Stieltjes integrals representing the fields in the infinite $\mathbf{x}$-plane have been replaced by sums for convenience of notation. The integral limit $L \rightarrow \infty$ may be taken after forming ensembleexpectation values, thereby avoiding the unwieldy notation of generalised integrals in the representation of individual realisations. 
It can be readily seen that an irrotational wave field which is driven by a given interior force can also be described by Equations (2.1)-(2.4), where $\hat{p}_{\mathbf{k}}$ in this case denotes an equivalent surface pressure which can be expressed in terms of the interior force field.

There is no restriction on the structure of the force field. In general, any interior force can be decomposed into two components, of which one contributes to the generation of linear surface waves, whereas the other component generates currents. Since we shall be concerned here only with interactions which affect the wave field, we may ignore the current-generating term.

Accordingly, any surface-wave interaction problem reduces to the harmonic oscillator Equation (2.1), where the coupling is described by the equivalent pressure field $\hat{p}$.

For the formal treatment of wave-interaction problems it is convenient to transform to standard wave variables (cf., Peierls, 1955; Hasselmann, 1968), which may be defined in the present case as

$$
a_{\mathbf{k}}^{ \pm}=\frac{1}{2}\left(\zeta_{\mathbf{k}} \pm \frac{i}{\sigma} \frac{\partial \zeta_{\mathbf{k}}}{\partial t}\right)
$$

Substitution in Equation (2.1) yields the two first-order equations

$$
\frac{\partial a_{\mathbf{k}}^{s}}{\partial t}+i s \sigma a_{\mathbf{k}}^{\mathbf{s}}=-\frac{i s \sigma}{2 g \varrho_{w}} \hat{p}_{\mathbf{k}} \quad(s= \pm)
$$

in which the two homogeneous solutions of (2.1), representing waves propagating in the positive and negative $k$-directions, have been separated into the two solutions $a_{\mathbf{k}}^{s}=\alpha_{\mathbf{k}}^{s} e^{-i s \sigma t}, \alpha_{\mathrm{k}}^{s}=$ const, of the separate equations $s= \pm$ in (2.6). The wave variables satisfy the reality conditions $a_{\mathrm{k}}^{s}=\left(a_{-\mathrm{k}}^{-s}\right)^{*}$.

In terms of $a_{\mathbf{k}}^{s}$, the surface displacement becomes

$$
\check{\zeta}=\sum_{\mathbf{k}}\left(a_{\mathbf{k}}^{+}+a_{\mathbf{k}}^{-}\right) e^{i \mathbf{k} \cdot \mathbf{x}} .
$$

Assuming that the wave components are statistically orthogonal, the mean-square surface displacement is then given by

$$
\left\langle\zeta^{2}\right\rangle=2 \sum_{\mathbf{k}}\left\langle\left|a_{\mathbf{k}}^{+}\right|^{2}\right\rangle=\sum_{\mathbf{k}} E_{\mathbf{k}}
$$

where $E_{\mathbf{k}}$ denotes the wave spectrum. We shall also use interchangeably with the discrete notation the familiar continuous one- and two-dimensional spectra defined by

$$
\sum_{\mathbf{k}} E_{\mathbf{k}}=\iint E(\mathbf{k}) \mathrm{d} \mathbf{k}=\iint F(\sigma) s(\sigma, \theta) \mathrm{d} \sigma \mathrm{d} \theta=\int F(\sigma) \mathrm{d} \sigma
$$

where $s(\sigma, \theta)$ denotes the spreading factor with respect to the wave-propagation direction $\theta$, normalised such that $\int_{-\pi}^{+\pi} s(\sigma, \theta) \mathrm{d} \theta=1$. 
Multiplication of Equation (2.6) by $a_{-\mathbf{k}}^{-s}$ and addition of the complex conjugate yields the energy equation*

$$
\frac{\partial E_{\mathbf{k}}}{\partial t}=\frac{2 \sigma}{g \varrho_{w}} \operatorname{Im}\left\langle\hat{p}_{\mathbf{k}} a_{-\mathbf{k}}^{-}\right\rangle .
$$

The right-hand side of (2.9) may be recognised as the mean work done against the vertical orbital velocity of the wave component $\mathbf{k}$ by the pressure field $\hat{p}$ (of which only the component $\hat{p}_{\mathbf{k}}$ contributes).

We assume now that for a given interaction problem, $\hat{p}_{\mathbf{k}}$ is a prescribed functional of the wave field, $\hat{p}_{\mathbf{k}}=\hat{p}_{\mathbf{k}}\left(\ldots, a_{\mathbf{k}}^{s}, \ldots\right)$. The functional may include also a dependence on external fields, such as the atmospheric boundary layer, currents, turbulence in the ocean, etc., but these are irrelevant for the present and will not be included explicitly.

If the functional $\hat{p}_{\mathrm{k}}$ can be adequately represented by the first few terms of a Taylorseries expansion with respect to the wave variables $a_{\mathbf{k}}^{s}$, the coupling represents a weakinteraction problem which can be treated by standard perturbation techniques. Unfortunately, this approach is not applicable to processes involving locally strong interactions, such as white capping. However, a simple yet general technique for treating non-expansible interactions has been outlined by Hasselmann and Collins (1968) in an earlier analysis of a locally strong process, the dissipation of finite-depth gravity waves by turbulent bottom friction.

The basic hypothesis of the analysis is that the interactions, although strong locally are weak-in-the-mean. This implies that the change induced in the wave spectrum per unit wavelength or period is small, and that the Gaussian property of a linear wave field applies to lowest order. Although the interaction functional is non-expansible, the wave field itself may then be represented as a perturbation series:

$$
a_{\mathbf{k}}^{s}=a_{\mathbf{k}, 0}^{s}+a_{\mathbf{k}, 1}^{s}+a_{\mathbf{k}, 2}^{s}+\cdots,
$$

where $a_{\mathbf{k}, 0}^{s}=\alpha_{\mathbf{k}}^{s} e^{-i s \sigma t}$ is the zeroth-order free-wave solution with Gaussian statistics, and the terms $a_{\mathbf{k}, n}^{s}$ are of order $n$ in an appropriate perturbation parameter characterising the mean strength of the interaction. To lowest order, (2.9) may then be written

$$
\frac{\partial E_{\mathbf{k}}}{\partial t}=\frac{2 \sigma}{g \varrho_{w}} \operatorname{Im}\left\langle\hat{p}_{\mathbf{k}}\left(\ldots, a_{\mathbf{k}, 0}^{s}, \ldots\right) a_{-\mathbf{k}, 0}^{-}\right\rangle .
$$

It may be noted that for expansible wave-wave interaction problems, the lowest order expression for the source function on the right-hand side of (2.11) vanishes. In this case the leading term in the Taylor expansion of $\hat{p}_{\mathbf{k}}$ is normally quadratic in the wave amplitudes, so that the mean product in (2.11) is cubic, which vanishes for a Gaussian field. Hence the functional $\hat{p}_{\mathbf{k}}$ needs to be expanded to higher order. However,

* Following the established custom, we refer here to the variance spectrum of the surface displacement as the 'energy' spectrum. To convert later to genuine energy and momentum transfer rates, a factor $Q_{w} g$ has to be added. 
it is then no longer consistent to substitute the zeroth-order expressions for the wave components in the right-hand side of (2.11), and higher order terms of the wave field itself must also be evaluated. These complications do not arise in the case of nonexpansible interactions, as the lowest-order source term is normally nonzero; it is therefore sufficient to consider only the zeroth-order wave field.

To determine the correlation of $\hat{p}_{\mathbf{k}}$ with $a_{-\mathbf{k}, 0}^{-}$in (2.11), the wave field may be divided into two constituents, $A=A^{\prime}+a_{\mathrm{k}}^{+}$, where

$$
A=\left\{a_{\mathbf{k}_{1}}^{s_{1}}, \ldots a_{\mathbf{k}_{n-1}}^{s_{n-1}}, a_{\mathbf{k}_{n}}^{s_{n}}, a_{\mathbf{k}_{n+1}}^{s_{n}-1}, \ldots\right\}
$$

represents the complete wave field and

$$
A^{\prime}=\left\{a_{\mathbf{k}_{1}}^{s_{1}}, \ldots a_{\mathbf{k}_{n-1}}^{s_{n-1}}, 0, a_{\mathbf{k}_{n+1}}^{s_{n-1}}, \ldots\right\}
$$

represents the complete field minus the (infinitesimal) wave component $a_{\mathbf{k}_{\mathbf{n}}}^{s_{n}} \equiv a_{\mathbf{k}}^{+}$. Assuming that the pressure field is differentiable, it may then be written as

$$
\hat{p}_{\mathbf{k}}(A)=\hat{p}_{\mathbf{k}}\left(A^{\prime}\right)+\frac{\partial \hat{p}_{\mathbf{k}}}{\partial a_{\mathbf{k}}}\left(A^{\prime}\right) a_{\mathbf{k}}^{+}+\cdots
$$

which yields on substitution in Equation (2.11),

$$
\frac{\partial E_{\mathbf{k}}}{\partial t}=\frac{2 \sigma}{g \varrho_{w}} \operatorname{Im}\left\{\left\langle\hat{p}_{\mathbf{k}}\left(A^{\prime}\right) a_{-\mathbf{k}}^{-}\right\rangle+\left\langle\frac{\partial \hat{p}^{\mathbf{k}}}{\partial a_{\mathbf{k}}^{+}}\left(A^{\prime}\right) \cdot\left|a_{\mathbf{k}}^{+}\right|^{2}\right\rangle\right\} .
$$

(The subscript zero denoting the zeroth-order free-wave field has now been dropped.)

Since all wave components are statistically independent, $A^{\prime}$ and $a_{\mathbf{k}}^{+}$represent statistically independent variables. Hence the expectation values of the quadratic products in (2.12) may be applied separately to each term. The first product vanishes, since $\left\langle a_{-\mathbf{k}}^{-}\right\rangle=0$, and the second term yields

$$
\frac{\partial E_{\mathbf{k}}}{\partial t}=-\gamma E_{\mathbf{k}}
$$

where

$$
\gamma=-\frac{\sigma}{g \varrho_{w}} \operatorname{Im}\left\langle\frac{\partial \hat{p}_{\mathbf{k}}}{\partial a_{\mathbf{k}}^{+}}(A)\right\rangle
$$

The field $A^{\prime}$ in (2.12) has been replaced again in (2.14) by the complete field $A$, since the fields differ only by an infinitesimal quantity.

The quasi-linear form (2.13) holds generally for any differentiable interaction process which is weak-in-the-mean and for which $\left\langle\partial \hat{p}_{\mathbf{k}} / \partial a_{\mathbf{k}}^{+}\right\rangle \neq 0$. For example, source functions of this form have been derived for various theories of the linear interaction between surface waves and the turbulent atmospheric boundary layer, the damping of finite-depth waves by turbulent bottom friction, and 'parametric interactions' between waves and currents or waves and turbulence (cf., Phillips, 1966; Hasselmann, 
1968). Jeffreys' (1924) theory of wave generation by air-flow separation, although presumably strongly nonlinear in the wave field, should also yield a source function of this form. However, it may be expected that in contrast to Jeffreys' expression for monochromatic waves, the factor $\gamma$ for a random wave field will be a function of the overall wave statistics.

In the following we shall attempt to evaluate $\gamma$ for the particular case in which the pressure field $\hat{p}_{\mathbf{k}}$ is associated with white capping.

\section{The Damping Factor $\gamma$}

An explicit determination of the white-cap pressure field $\hat{p}$ would require consideration of the internal fluid-dynamical structure of a breaking wave. Unfortunately, a suitable white-cap model on which such calculations could be based does not exist. Even the considerable work which has been devoted to the simpler question of the limiting wave form has focussed on special cases, such as the periodic progressive wave or standing wave, and not arbitrary time-dependent wave forms as encountered in a random wave field. It appears unlikely that the highly complex problem of deriving a general functional relationship between the equivalent white-cap pressure field $\hat{p}$ and the properties of the wave field near the wave crest can be solved in the near future. Accordingly, we shall attempt to avoid this difficulty by determining certain properties of the factor $\gamma$ which are insensitive to the details of the white-capping process. It is found, for example, that the frequency dependence of $\gamma$ can be deduced alone from the hypothesis that the space-time scales of the white caps are small compared with the wavelengths and periods of the wave components to which $\gamma$ applies. The remaining free factor is constant, except for a directional dependence, for a given wave state. It cannot be evaluated within the framework of the present theory alone, but we shall attempt a determination later based on (occasionally speculative) considerations of the overall energy balance of the spectrum (Sections 4, 5).

Basic for the following analysis will be the assumption that the pressure pulse characterising the interactions in a given white cap is a narrow distribution in space and time concentrated around a point $\tilde{\mathbf{x}}, \tilde{t}$ defined by the position and time of the maximum surface displacement, $\partial \zeta / \partial x_{j}=\partial \zeta / \partial t=0$ at $\mathbf{x}=\tilde{\mathbf{x}}, t=\tilde{t}$. It will be convenient to characterise the pressure pulse by its moments about $\tilde{\mathbf{x}}, \tilde{z}$;

$$
\left\{\begin{array}{c}
m_{0} \\
m_{t} \\
m_{i} \\
\vdots
\end{array}\right\}=\iiint \mathrm{d} \tilde{\mathbf{x}} \mathrm{d} t \hat{p}(\tilde{\mathbf{x}}, t)\left\{\begin{array}{c}
1 \\
t-\tilde{t} \\
x_{i}-\tilde{x}_{i} \\
\vdots
\end{array}\right\}
$$

(To avoid introducing a new symbol, $\hat{p}$ is used here to denote the pressure pulse of a single white cap; later $\hat{p}$ will again represent the entire pressure field resulting from the superposition of all white-cap pressure pulses.)

In order to express $\gamma$ more easily in terms of the pressure moments, the quasistationarity of the wave field may be invoked to introduce a time average in Equation 
(2.14) prior to the ensemble average,

$$
\gamma=-\frac{\sigma}{g \varrho_{w}} \operatorname{Im}\left\langle\frac{1}{\tau} \int_{-\tau / 2}^{\tau / 2} \frac{\partial \hat{p}_{\mathbf{k}}}{\partial a_{\mathbf{k}}^{+}} \mathrm{d} t\right\rangle
$$

where $\tau$ is a (large) time period encompassing the relatively short interval in which the white cap occurs. A spatial average is already implicit in the Fourier component. Hence the damping coefficient may be written in the form of a space-time integral over the pressure pulse

$$
\gamma=-\frac{\sigma}{g \varrho_{w}} \operatorname{Im}\left\langle\frac{1}{\tau L^{2}} \int_{-\tau / 2}^{\tau / 2} \int_{-L / 2}^{L / 2} \int_{-2} \mathrm{~d} \mathbf{x} \mathrm{d} t \frac{\partial \hat{p}}{\partial a_{\mathbf{k}}^{+}}(\mathbf{x}, t) e^{-i \mathbf{k} \cdot \mathbf{x}}\right\rangle .
$$

We assume now that the pressure pulse of a given white cap can be expressed as a function of various local properties of the wave field $\tilde{\beta}_{1}, \tilde{\beta}_{2}, \ldots$ at the wave crest, for example, the surface displacement $\tilde{\zeta}$, the acceleration $\partial^{2} \tilde{\zeta} / \partial t^{2}$, the second derivatives $\partial^{2} \tilde{\zeta} / \partial x_{i} \partial x_{j}, \partial^{2} \tilde{\zeta} / \partial x \partial \mathrm{t}$, etc. (Here as in the following, the tilde denotes values taken at $\tilde{x}, \tilde{t}$.) Then $\hat{p}(x, t)=\not p\left(\mathbf{x}-\tilde{\mathbf{x}}, t-\tilde{t} ; \tilde{\beta}_{1}, \tilde{\beta}_{2}, \ldots\right)$ so that

$$
\frac{\partial \hat{p}}{\partial a_{\mathbf{k}}^{+}}=-\frac{\partial \not h}{\partial x_{j}} \frac{\partial \tilde{x}_{j}}{\partial a_{\mathbf{k}}^{+}}-\frac{\partial \not h}{\partial t} \frac{\partial \tilde{t}}{\partial a_{\mathbf{k}}^{+}}+\frac{\partial \not h}{\partial \tilde{\beta}_{j}} \frac{\partial \tilde{\beta}_{j}}{\partial a_{\mathbf{k}}^{+}} .
$$

We may regard $\tilde{\mathbf{x}}, \tilde{t}$ and the parameters $\tilde{\beta}_{j}$ as being defined in terms of the linear field representation (2.7), with $a_{\mathbf{k}}^{s}=a_{\mathbf{k} 0}^{s}$. Because of nonlinear corrections, $\tilde{\mathbf{x}}, \tilde{t}$ will then not denote the true position and time of the maximal surface displacement, nor will the parameters $\widetilde{\beta}_{j}$ represent identically the true displacement, acceleration, etc. at $\tilde{x}, \tilde{t}$. However, provided the differences between the true crest variables and their equivalent linear forms are not so great that the two sets of variables cannot be uniquely related, the linearised variables may still be used as a consistent set of parameters characterising the properties of the pressure pulse - although it must then be borne in mind that the physical interpretation of these parameters in terms of wave properties at the wave crest is only approximate.

For a linear wave field, the variables $\tilde{\beta}_{j}$ are of the general form

$$
\tilde{\beta}_{j}=\sum_{\mathbf{k}, s} B_{j}^{s} a_{\mathbf{k}}^{s}(\tilde{t}) e^{i \mathbf{k} \cdot \tilde{\mathbf{x}}}
$$

where $B_{j}^{s}(\mathbf{k})$ is a transfer function. Since the wave variable refers here to the zerothorder solution $a_{\mathbf{k}}^{s}(t)=\alpha_{\mathbf{k}}^{s} e^{-i s \sigma t}$, the value of $a_{\mathbf{k}}^{s}$ at $\tilde{t}$ may be written $a_{\mathbf{k}}^{s}(t) e^{i s \sigma(t-\tilde{t})}$, and the derivative of $\tilde{\beta}_{j}$ with respect to $a_{\mathrm{k}}^{+}(t)$ in (3.2) becomes

$$
\frac{\partial \tilde{\beta}_{j}}{\partial a_{\mathbf{k}}^{+}}=B_{j}^{+} \operatorname{cxp}[i \sigma(t-\tilde{t})+i \mathbf{k} \cdot \tilde{\mathbf{x}}]+\frac{\partial \tilde{\beta}_{j}}{\partial \tilde{x}_{l}} \frac{\partial \tilde{x}_{l}}{\partial a_{\mathbf{k}}^{+}}+\frac{\partial \tilde{\beta}_{j}}{\partial \tilde{t}} \frac{\partial \hat{t}}{\partial a_{\mathbf{k}}^{+}} .
$$

Thus the derivative $\partial \tilde{\beta}_{j} / \partial a_{\mathbf{k}}^{+}$in (3.2) may be expressed in terms of the remaining derivatives $\partial \tilde{x}_{l} / \partial a_{\mathbf{k}}^{+}$and $\partial \tilde{t} / \partial a_{\mathbf{k}}^{+}$. These in turn may be determined from (3.3) by sub- 
stituting for $\tilde{\beta}_{j}$ the first derivatives $\partial \tilde{\zeta} / \partial x_{j}$ and $\partial \tilde{\zeta} / \partial t$, for which the left-hand side of the equation vanishes by definition of the maximal surface displacement. Explicitly, (3.3) yields in this case

$$
\begin{aligned}
& \frac{\partial}{\partial a_{\mathbf{k}}^{+}}\left(\frac{\partial \tilde{\zeta}}{\partial x_{i}}\right)=0=i k_{i} \exp [i \sigma(t-\tilde{t})+i \mathbf{k} \cdot \tilde{\mathbf{x}}]+\frac{\partial^{2} \tilde{\zeta}}{\partial x_{i} \partial x_{l}} \frac{\partial \tilde{x}_{l}}{\partial a_{\mathbf{k}}^{+}}+\frac{\partial^{2} \tilde{\zeta}}{\partial x_{i} \partial t} \frac{\partial \tilde{t}}{\partial a_{\mathbf{k}}^{+}} \\
& \frac{\partial}{\partial a_{\mathbf{k}}^{+}}\left(\frac{\partial \tilde{\zeta}}{\partial t}\right)=0=-i \sigma \exp [i \sigma(t-\tilde{t})+i \mathbf{k} \cdot \tilde{\mathbf{x}}]+\frac{\partial^{2} \tilde{\zeta}}{\partial t \partial x_{l}} \frac{\partial \tilde{x}_{1}}{\partial a_{\mathbf{k}}^{+}}+\frac{\partial^{2} \tilde{\zeta}}{\partial t^{2}} \frac{\partial \tilde{t}}{\partial a_{\mathbf{k}}^{+}} \text {. }
\end{aligned}
$$

The solutions of (3.4) are of the form

$$
\left(\begin{array}{c}
\frac{\partial \tilde{x}_{i}}{\partial a_{\mathbf{k}}^{+}} \\
\frac{\partial \tilde{t}}{\partial a_{\mathbf{k}}^{+}}
\end{array}\right)=\left(\begin{array}{c}
i X_{i} \\
i T
\end{array}\right) \exp [i \sigma(t-\tilde{t})+i \mathbf{k} \cdot \tilde{\mathbf{x}}]
$$

with real $X_{i}$ and $T$.

Substituting (3.2), (3.3) and (3.5) in (3.1), the expression for $\gamma$ becomes

$$
\begin{aligned}
\gamma= & -\frac{\sigma}{g \varrho_{w}} \operatorname{Im}\left\langle\frac { 1 } { \tau L ^ { 2 } } \int \int \int \mathrm { d } t \mathrm { d } \mathbf { x } \left\{-i X_{i} \frac{\partial \not p}{\partial x_{i}}-i T \frac{\partial \not}{\partial t}+\frac{\partial \dot{p}^{\prime}}{\partial \tilde{\beta}_{j}} \times\right.\right. \\
& \left.\left.\times\left[B_{j}^{+}+i X_{i} \frac{\partial \tilde{\beta}_{j}}{\partial \tilde{x}_{i}}+i T \frac{\partial \tilde{\beta}_{j}}{\partial \tau}\right]\right\} \exp [i \sigma(t-\tilde{t})-i \mathbf{k} \cdot(\mathbf{x}-\tilde{\mathbf{x}})]\right\rangle .
\end{aligned}
$$

Integrating the first two terms by parts, expanding the exponential about $\tilde{\mathbf{x}}, \tilde{t}$ and retaining only the lowest order pressure moment for each term, one obtains

$$
\begin{aligned}
\gamma= & \frac{\sigma}{g \varrho_{w}} N\left\{k_{i} k_{j}\left\langle X_{i} m_{j}\right\rangle+\sigma^{2}\left\langle T m_{t}\right\rangle+B_{j}^{+}\left[k_{l}\left\langle\frac{\partial m_{l}}{\partial \tilde{\beta}_{j}}\right\rangle-\sigma\left\langle\frac{\partial m_{t}}{\partial \tilde{\beta}_{j}}\right\rangle\right.\right. \\
& \left.\left.-\left\langle\left(X_{i} \frac{\partial \tilde{\beta}_{j}}{\partial \tilde{x}_{i}}+T \frac{\partial \tilde{\beta}_{j}}{\partial \tilde{t}}\right) \frac{\partial m_{0}}{\partial \tilde{\beta}_{j}}\right\rangle\right]\right\} .
\end{aligned}
$$

In Equation (3.7) we have changed back to the original definition of $\gamma$ and $\hat{p}$ in terms of the complete field of white caps, rather than a single white cap, by summing over all white caps. This is expressed by the replacement of the factor $1 / \tau L^{2}$ in (3.6) by the number of white caps $N$ per unit surface area and unit time.

It may be helpful to retrace briefly the origin of the various terms in (3.7). Except for a factor, $\gamma$ represents the (infinitesimal) part of the pressure pulse $\hat{p}$ which is correlated in quadrature with the wave component $a_{\mathbf{k}}^{+}$. This was determined in Section 2 by considering the infinitesimal variation $\delta \hat{p}_{\mathbf{k}}$ induced by the component $a_{\mathbf{k}}^{+}$relative to the pressure $\hat{p}_{\mathbf{k}}$ for the same wave field without the spectral component $\mathbf{k}$. For the particular pulse representation considered here, $\delta \hat{p}_{\mathbf{k}}$ consists of the changes induced by the shift $\delta \tilde{\mathbf{x}}$ and $\delta \tilde{t}$ of the reference point, which affects the evaluation of the moments 
(first and second terms in the right-hand side of (3.7)), and the changes due to variations $\delta \beta_{j}$ of the local wave parameters at the reference point (proportional to $B_{\mathbf{j}}^{+}$). The latter can be divided further into the variations of $\beta_{j}$ for fixed $\hat{\mathbf{x}}, \mathfrak{t}$ (first two terms and the changes in $\beta_{j}$ caused by varying $\hat{\mathbf{x}}$ and $\tilde{t}$ (third term).

Up to this point, no approximations have been introduced other than that the white-cap scales are small compared with $k^{-1}$ or $\sigma^{-1}$. To reduce (3.7) further, we may estimate the orders of magnitude of the individual terms in (3.7) from scale considerations.

For frequencies beyond the wind-sea peak, the wave spectrum may be represented fairly well by the Phillips' $(1958,1966)$ form

$$
E(\mathbf{k})=\alpha / 2 k^{-4} s(\theta)
$$

or

$$
E(\sigma)=\alpha g^{2} \sigma^{-5}
$$

For this distribution, the rms values of the quadratic derivatives in (3.4) are governed by the high-frequency range of the spectrum and depend explicitly on a cut-off frequency $\sigma_{s}$ or wave number $k_{s}=\sigma_{s}^{2} / g$. The orders of magnitude of the transfer functions in (3.5) may then be estimated as $X_{i}=\sigma 0\left(\left(\sigma_{s} k_{s} \tilde{\zeta}\right)^{-1}\right), T=\sigma 0\left(\sigma_{s}^{-2} \tilde{\zeta}^{-1}\right)$. We assume that the relevant cut-off for rms values evaluated at $\tilde{\mathbf{x}}, \tilde{t}$ is determined by the white-cap scale, and that the same scale may also be used in relating the moments of the whitecap pressure pulse, $m_{0}: m_{i}: m_{t}=0\left(1: k_{s}^{-1}: \sigma_{s}^{-1}\right)$. If furthermore $\partial / \partial \tilde{\beta}_{j}=0\left(1 / \beta_{j}\right)$, the largest contributions in (3.7) are found to be

$$
\left\langle\left(X_{i} \frac{\partial \tilde{\beta}_{j}}{\partial \tilde{x}_{i}}+T \frac{\partial \tilde{\beta}_{j}}{\partial \tilde{\tau}}\right) \frac{\partial m_{0}}{\partial \tilde{\beta}_{j}}\right\rangle=\sigma 0\left(m_{0} \sigma_{s}^{-1} \tilde{\zeta}^{-1}\right)
$$

(for those $\beta_{j}$ for which the rms values of $\partial \tilde{\beta}_{j} / \partial x_{i}$ or $\partial \tilde{\beta}_{j} / \partial t$ are governed by the shortwave range of the spectrum - otherwise the term is of smaller magnitude) and the term $B_{j}^{+} \sigma\left\langle\partial m_{t} / \partial \tilde{\beta}_{j}\right\rangle$ for the case $\beta_{j} \equiv \tilde{\zeta}$,

$$
\sigma\left\langle\partial m_{t} / \partial \tilde{\zeta}\right\rangle=\sigma 0\left(m_{0} \sigma_{s}^{-1} \tilde{\zeta}^{-1}\right)
$$

Both terms yield to lowest order

$$
\gamma=\eta \sigma^{2}
$$

where the factor $\eta$ is a function of the wave-propagation direction and the overall (integral) statistical properties of the wave field; it is constant for a given wave state. Equation (3.9) applies for $\sigma \ll \sigma_{s}$, representing the first term in an expansion of the general form $\gamma=\eta \sigma^{2}+\eta_{1} \sigma^{3} / \sigma_{s}+\eta_{2} \sigma^{4} / \sigma_{s}^{2}+\cdots$.

To proceed further and evaluate the coefficients $\eta, \eta_{1}, \eta_{2}, \ldots$ would require consideration of the structure of the pressure pulse for a given white-cap model. As has been pointed out, the development of such a model is not an easy step and will not be attempted here. However, even if this were feasible, it is questionable whether knowl- 
edge of the precise functional dependence of the coefficient $\eta$ on the wave statistics would be of much value in practice. It may be anticipated that $\eta$ is a rather sensitive function of the rms surface acceleration and other properties of the wave field. In this case, the wave field will normally be in a 'saturated' state in which relatively minor changes in the wave statistics can produce large changes in the dissipation rate. Rather than attempting to calculate $\eta$ from the wave statistics alone, which would require accurate knowledge of the wave spectrum, it may then be more practicable to establish the absolute level of the dissipation function indirectly from consideration of the overall energy and momentum balance of the wave spectrum.

Clearly, this alternative approach requires that the remaining processes governing the energy balance are reasonably well understood. To date, this is only partially the case. However, a number of field investigations of wave growth (Snyder and Cox, 1966; Barnett and Wilkerson, 1967; Mitsuyasu, 1968, 1969; Mitsuyasu et al., 1971; Ross et al., 1970; Schule et al., 1971; and Hasselmann et al., 1973) have identified several basic features of the spectral energy balance, making it meaningful to at least investigate the form of the proposed wave-breaking source function

$$
\left(\frac{\partial E(\mathbf{k})}{\partial t}\right)_{\text {wave breaking }} \equiv S_{w b}=-\eta \sigma^{2} E(\mathbf{k})
$$

in relation to existing concepts of the energy balance.

It is found that (3.10) fits naturally into the existing framework. Thus encouraged, we shall attempt to complete the picture of the energy balance by proposing a simple expression for the least known source function $S_{\mathrm{in}}$, representing the energy input by the wind. With the energy balance completed, $\eta$ can then be determined. However, it must be stressed that in the present stage this last step is speculative and will need to be confirmed or modified by more detailed field studies of the air-sea interaction processes. The argument is carried through completely here largely because we expect the method to remain applicable even if the input source function should need to be modified. Also, it is felt that despite these shortcomings the proposed set of source functions contains fewer hypotheses than involved in current wave-prediction schemes.

\section{The Energy and Momentum Balance of the Wave Spectrum}

Before considering the contribution of the wave-breaking source function to the spectral energy balance, we review briefly the general structure of the energy balance as derived from wave-growth studies, in particular from JONSWAP. We shall be concerned only with the one-dimensional energy-balance equation

$$
\frac{\partial F(\sigma)}{\partial t}+\bar{v} \frac{\partial F(\sigma)}{\partial x}=S \quad\left(\left\{\begin{array}{l}
\bar{v} \\
S
\end{array}\right\}=\int_{-\pi}^{\pi}\left\{\begin{array}{c}
v_{1} \\
S_{2}
\end{array}\right\} s(\sigma, \theta) \mathrm{d} \theta, \quad \mathbf{v}=\text { group velocity }\right)
$$


obtained by averaging the two-dimensional energy-balance equation

$$
\frac{\partial}{\partial t}(F s)+v_{i} \frac{\partial}{\partial x_{i}}(F s)=S_{2}(\sigma, \theta)
$$

over the wave propagation direction $\theta$, measured relative to the mean wind direction parallel to $x_{1}$. Analogous equations for the momentum balance may be obtained by multiplying (4.2) by the momentum/energy ratio $\mathbf{k} / \sigma$. (We remind again that a factor $\zeta_{w} g$ should be included in (4.2) to convert to genuine energy and momentum.)

The net source function $S$ can be divided generally into three constituents

$$
S=S_{\mathrm{in}}+S_{\mathrm{nl}}+S_{\mathrm{ds}}
$$

representing, respectively, the input by the wind, the nonlinear transfer across the spectrum by conservative wave-wave interactions (third-order resonant interactions), and dissipative processes (including non-conservative nonlinear interactions). Measurements of wavegrowth yield the net source function $S$ through (4.1). $S_{\mathrm{n} 1}$ can be computed, for a given spectrum, from the appropriate Boltzmann-integral transfer expressions (Hasselmann, 1962, 1963a, b). The source function $S_{\text {in }}$ has been determined from cross-spectral measurements of surface pressure and surface displacement (Dobson, 1971a; Elliott, 1972; Snyder, 1973), but these measurements have not been combined with wave-growth studies. There exists as yet no technique for the direct measurement of $S_{\mathrm{ds}}$.

An important result of JONSWAP was that the qualitative features of a growing wave spectrum could be largely explained in terms of the nonlinear energy transfer $S_{\mathrm{n} 1}$. A series of computations of $S_{\mathrm{n} 1}$ were made for various spectral shapes. These showed that for any flattish spectral distribution with a low-frequency cut-off, the nonlinear energy transfer tends to concentrate energy at frequencies slightly higher than the cut-off. The peak formed in this manner continues to grow at the same frequency until it becomes about as narrow as the spectral peak shown in Figure 1. At this stage, the nonlinear energy transfer shifts to slightly lower frequencies to the left of the peak. Consequently, the peak no longer grows but moves as a whole towards lower frequencies. A peak sharper than the distribution shown in Figure 1 is found to flatten again in addition to shifting. Thus the development of a pronounced spectral peak and the continuous movement of the peak towards lower frequencies may be explained as a self-stabilising feature of the nonlinear energy transfer. Consistent with this explanation, it was found that most of the observed wave growth $S$ on the forward face of the spectrum could be attributed to the nonlinear transfer $S_{\mathrm{n} 1}$ (Figure 1).

Qualitatively, these features are independent of the magnitude and distribution of $S_{\text {in }}$ and $S_{\mathrm{ds}}$. However, both the level of the wave spectrum and the rate at which the peak moves towards lower frequencies depend strongly on these source functions. Unfortunately, knowledge of $S$ and $S_{\mathrm{n} 1}$ from wave-growth studies alone is insufficient to determine $S_{\mathrm{in}}$ and $S_{\mathrm{ds}}$ separately from (4.3) without further information.

In the case of small fetches $\left(10^{2} \leqslant g x / U^{2} \lesssim 5 \times 10^{2}\right.$, corresponding to peak frequen- 
cies $\sigma_{m}$ in the range $5 \gtrsim\left(\sigma_{m} U / g\right) \gtrsim 3$, where $U$ is the wind speed $)$, the ambiguity is reduced by the additional side condition that the total momentum transfer $\tau_{w}$ from the wind to the waves cannot exceed the total momentum transfer $\tau$ across the air-sea interface. The latter quantity is known from atmospheric boundary-layer measurements, $\tau=$ $=\varrho_{a} c_{d} U^{2}$, where $\varrho_{a}$ is the density of air and - for neutral conditions - the drag coefficient $c_{d}=(1.01-1.5) \times 10^{-3}$ (cf., Hasse, 1970; Smith, 1970; Pond et al., 1971; Brocks and Krügermeyer, 1972; Dunckel et al., 1974, and others).

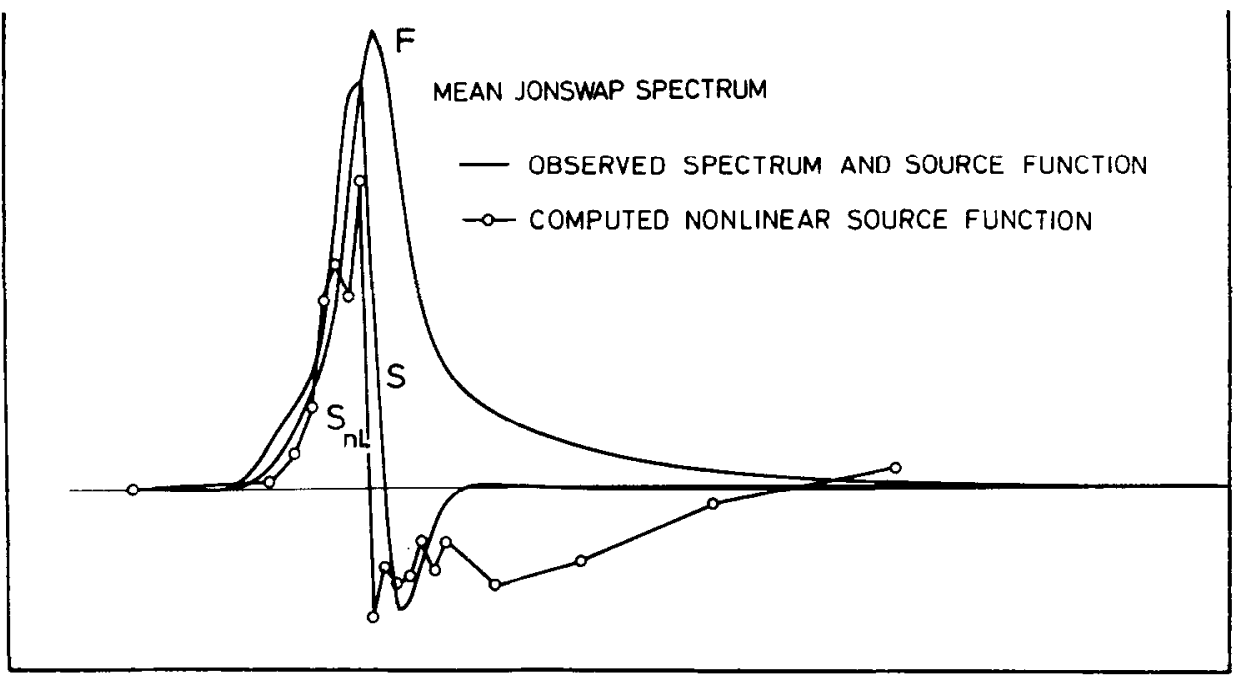

Fig. 1. Wave spectrum $F$, net source function $S$ and computed nonlinear transfer $S_{I 1}$ for fetchlimited spectra according to JONSWAP (from Hasselmann et al., 1973). Frequency and ordinate scales are arbitrary.

For small fetches, numerical integration of the net momentum $\tau_{\mathrm{nl}}^{\mathrm{hf}}$ gained by nonlinear interactions in the positive high-frequency lobe of $S_{\text {nI }}$ (i.e., the high-frequency region beyond the cross-over of $S_{\mathrm{n} 1}$ from negative to positive values, cf. Figure 1) generally yield values - although scattered - of the same order as $\tau$. The momentum $\tau_{n \mathrm{l}}^{\mathrm{hf}}$ is transferred to the high frequencies from the negative lobe of $S_{\mathrm{n} 1}$ near the spectral peak. (Part of the momentum from the central region is also transferred across the forward face to lower frequencies, causing the shift of the peak. The integrated momentum transfer rates of the positive low-frequency, negative mid-frequency and positive high-frequency lobes of $S_{\mathrm{n} 1}$ yield values in the ratio $1:-4: 3$, respectively; the corresponding energy ratios are $3:-5: 2$ ). The simplest way to complete the energy balance is then to assume that the negative central lobe of $S_{\mathrm{n} 1}$ is largely balanced by the input from the wind, and the positive high-frequency lobe by dissipation, with no overlap between $S_{\mathrm{in}}$ and $S_{\mathrm{ds}}$. The remaining low-frequency positive lobe of $S_{\mathrm{nl}}$ yields the wave growth. This solution is characterised by requiring a minimal momentum input $\tau_{w}$ from the wind, since any overlap of $S_{\mathrm{in}}$ and $S_{\mathrm{ds}}$ must yield higher 
absolute values of each source function separately in order to maintain the balance $S_{\mathrm{in}}+S_{\mathrm{ds}}=S-S_{\mathrm{nI}}$ (with given $S$ and $S_{\mathrm{nI}}$ ). In the minimal- $\tau_{w}$ case, the momentum dissipated in the high-frequency region of the spectrum, the momentum imparted to the waves by the wind in the central region of the spectrum and the nonlinear momentum transfer from medium frequencies to high frequencies are all approximately equal. Since $\tau_{\mathbf{n} l}^{\mathbf{h f}} \approx \tau$, it follows then that the minimal- $\tau_{w}$ solution already accounts for most of the momentum transfer across the air-sea interface and must therefore lie rather close to the true solution (cf., Hasselmann et al., 1973, Figure 2.22). Dobson (1971a) also found $\tau_{w} \approx \tau$ for small fetches. Elliott's (1972) estimates of $\tau_{w}$ are of the same order, but somewhat smaller. Contrary to these authors, Snyder (1973) finds that $\tau_{w}$ accounts for only a few percent of $\tau$.

Unfortunately, the minimal- $\tau_{w}$ solution is not consistent with the proposed frequency dependence (3.10) of the dissipation function. We suggest as explanation that for very small fetches, laminar viscous dissipation (Section 5) rather than wave breaking is the primary dissipation mechanism - which finds some support through the visual observation that white caps normally occur only at finite fetches. In the range $5 \times 10^{2} \lesssim g x / U^{2} \lesssim 10^{4}$, corresponding to peak frequencies $3 \gtrsim \sigma_{m} U / g \gtrsim 1.2$, the computed values of $\tau_{\mathrm{nl}}^{\mathrm{hf}}$ are almost an order of magnitude lower than $\tau$, and an energy balance with overlapping source functions $S_{\mathrm{in}}$ and $S_{\mathrm{ds}}$ is permissible. With $S_{\mathrm{ds}}$ given by (3.10), an energy balance of the general form shown in Figure 2 results. Here only 0.4 of the momentum $\tau_{w}$ imparted to the wave field is dissipated in the region of the positive high-frequency lobe of $S_{\mathrm{n} 1}$, whereas $0.5 \tau_{w}$ is dissipated at lower frequencies.

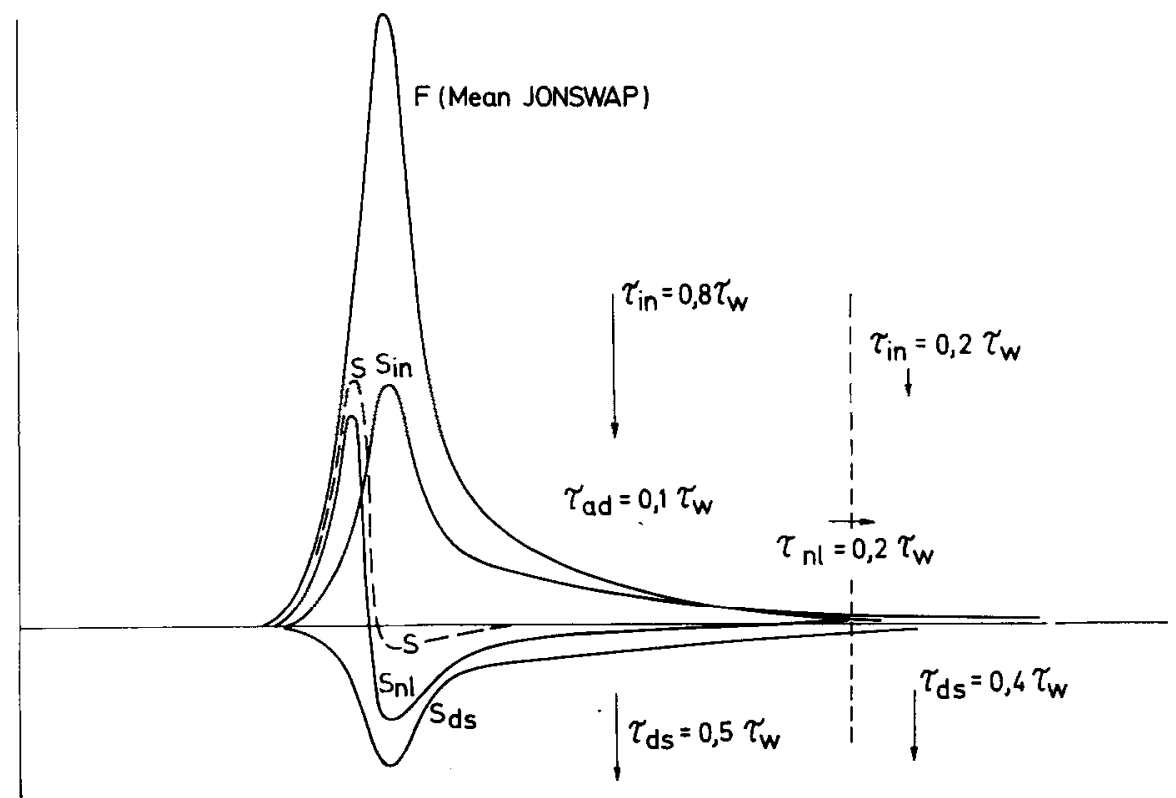

Fig. 2. Energy balance (schematic) for fetch-limited spectra in the fetch range $5 \times 10^{2} \lesssim g x / U^{2} \lesssim$ $\lesssim 10^{4}\left(3 \gtrsim \sigma_{m} U / g \gtrsim 1.2\right)$. Frequency and ordinate scales are arbitrary. 
The remaining $0.1 \tau_{w}$ is responsible for the wave growth and is therefore balanced by the advection $\tau_{\mathrm{ad}}$ of wave momentum. The total momentum input $\tau_{w}$ computed for this solution is of order $(0.5-1.0) \tau$.

For a fully developed Pierson-Moskowitz (1964) spectrum $\left(5 \times 10^{4} \lesssim g x / U^{2}\right.$, $\sigma_{m} U / g \approx 0.9$ ), an energy balance of the form shown in Figure 3 appears probable. The Pierson-Moskowitz spectrum is less sharply peaked (by a factor of 3 ) than the mean JONSWAP spectrum for finite fetches, and as a consequence, the low-frequency

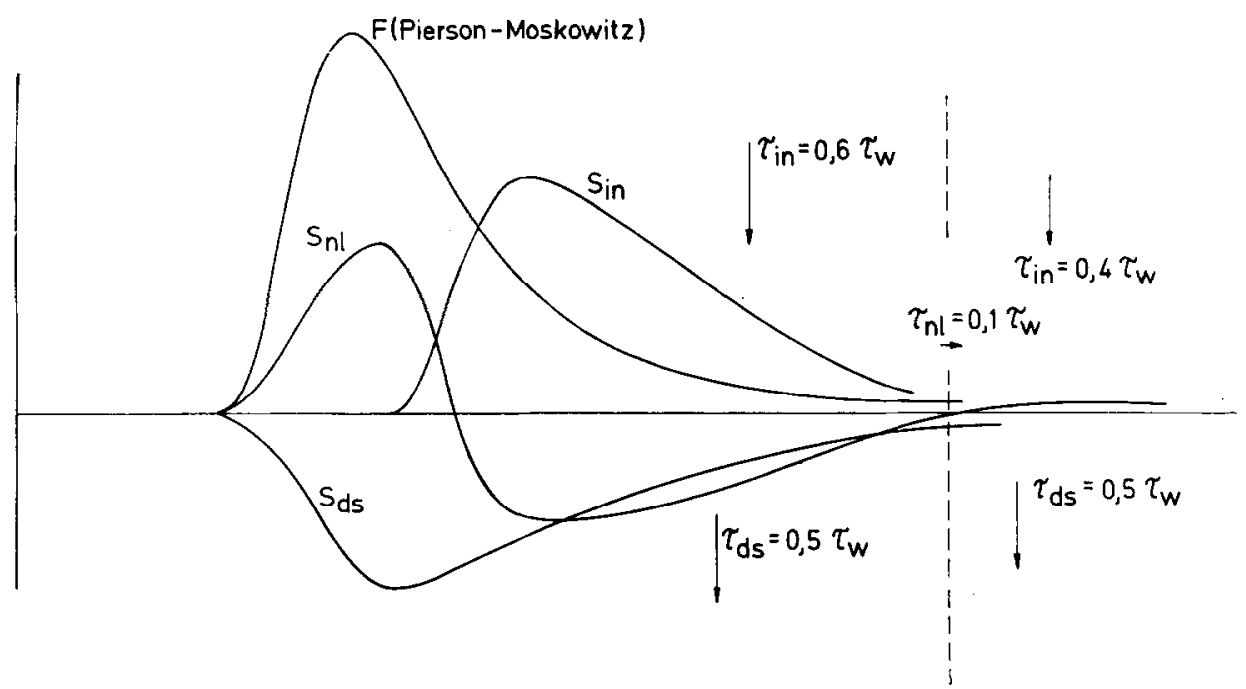

Fig. 3. Energy balance (schematic) for a fully developed Pierson-Moskowitz (1964) spectrum. Frequency and ordinate scales arbitrary.

positive lobe of $S_{\mathrm{n} 1}$ lies directly beneath the spectral peak, rather than on the forward face. It has been assumed, in accordance with most wave generation theories, that $S_{\mathrm{in}}$ approaches zero for $\sigma U / g<1$, so that $S_{\mathrm{in}}$ is shifted further to high frequencies than in the limited-fetch case. The dissipation balances the positive lobe of $S_{n 1}$ at the peak, most of the input to the right of the peak, and the second positive lobe of $S_{\mathrm{n} 1}$ at high frequencies. The momentum input $\tau_{w}$ is again found to be of order $(0.5-1.0) \tau$.

Although significant differences exist in the proposed energy balances at very small, intermediate and very large fetches, in all cases the resulting momentum transfer to the waves accounts for most of the air-sea momentum transfer. The same result can be inferred independently of the structure of the dissipation function if the input source function is of the general form

$$
S_{\mathrm{in}}=\beta E(\mathbf{k})
$$

where the growth factor $\beta$ is a function of $\mathbf{k}$ and $\mathbf{U}$ which is independent of the rest of the spectrum. An input source function of this form is characteristic for a linear feedback mechanism of wave generation, as has been frequently proposed (e.g., 
Jeffreys*, 1924; Miles, 1957; Phillips, 1966; Hasselmann, 1967, 1968; Davis, 1969, 1970; Long, 1971; Townsend, 1972).

It follows from (4.4) that a given frequency range of the spectrum will contribute the same energy and momentum input to the wave field at different fetches if the level of the spectrum remains constant. This is approximately the case for the highfrequency equilibrium range.

Consider now the frequency range $\sigma>\sigma_{0}$, where $\sigma_{0}$ is the peak frequency for some small fetch value, $g x / U^{2}=10^{2}$, say. At this fetch, the momentum input to the wave field in the range $\sigma>\sigma_{0}$ is computed from the minimal $\tau_{w}$-solution to be approximately $\tau$. However, at larger fetches, the range $\sigma>\sigma_{0}$ will also contribute approximately the same momentum input to the wave field, so that $\tau_{w} \approx \tau$ also at large fetches. In fact, since there is also some contribution to the momentum input in the range $\sigma_{m}<\sigma<\sigma_{0}$ at larger fetches, the equilibrium constant $\alpha$ must decrease slowly with fetch - as indeed observed (cf., Mitsuyasu, 1968; Hasselmann et al., 1973).

A simple expression for $S_{\text {in }}$ of the form (4.4) which yields $\tau_{w} \approx \tau$ for both small and large fetches and exhibits the general frequency dependence required by Figures 2 and 3 is given by **

$$
S_{\text {in }}= \begin{cases}\frac{\varrho_{a}}{\varrho_{w}}(\sigma \cos \theta-g / U) & \text { for } \sigma \cos \theta \geqslant g / u \\ 0 & \text { for } \sigma \cos \theta<g / u\end{cases}
$$

where $\theta$ is measured relative to the mean wind direction $\theta=0$.

It is assumed in (4.5) that $S_{\text {in }}=0$ for waves with phase velocities greater than the wind speed, although both laboratory evidence (cf., Harris, 1966; Lai and Shemdin, 1971) and field data (Dobson, 1971b, Volkoz and Mordukhovich, 1971) indicate that there may be a weak feedback of energy from these waves to the wind. The Expression (4.5) should be regarded only as a reasonable fit of an input function of the form (4.4) to the existing fetch-limited data, with a plausible extrapolation to the fully developed case. Further direct measurements of the input function $S_{\text {in }}$ are needed to resolve the discrepancies between existing surface-pressure measurements and to test whether the general linear form (4.4) and the extrapolation of (4.5) to the fully developed case are indeed valid.

\section{The Coefficient $\eta$}

Given an input source function $S_{\text {in }}$ of the form (4.5) and the computed nonlinear transfer $S_{\mathrm{n} 1}$, the coefficient $\eta$ in the dissipation source function can now be determined

* Jeffreys' flow-separation model should really be excluded, as it is presumably nonlinear if applied to a random field; the growth factor $\beta$ in (4.4) is than dependent on the rest of the wave field (cf. Section 2).

** Most input source functions used in present wave-prediction models are several factors larger than (4.5). They are based on earlier wave-growth studies in which the dominant contribution of nonlinear interactions to the growth rates on the forward face of the spectrum had not been recognised. Consequently, they yield $\tau_{w}$ values considerably grcater than the permissible upper limit (cf., Snyder and Cox, 1966). 
from the energy balance. For this purpose, we consider the high-frequency, equilibrium range of the spectrum for which $S=0$, so that $S_{\mathrm{ds}}=-S_{\mathrm{in}}-S_{\mathrm{n} 1}$. Specifically, we regard as high-frequency the spectral region corresponding to the third, positive lobe of $S_{\mathrm{n} 1}$, i.e., $\sigma>2.5 \sigma_{m}$. It is convenient to define the equilibrium condition in terms of the momentum integrals, which can be related to the known momentum flux $\tau$ across the air-sea interface and are insensitive to details of the spectral shape. The following calculations are based on a $\cos ^{2} \theta$ spreading factor; $\eta$ is assumed independent of $\theta$.

The total momentum gain due to nonlinear transfer in the high-frequency lobe is given by (Hasselmann et al., 1973)

$$
\tau_{\mathrm{nl}}^{\mathrm{hf}}=\lambda \varrho_{w} \alpha^{3}\left(g / \sigma_{m}\right)^{2}
$$

where the non-dimensional factor $\lambda$ depends only on the shape but not the energy and frequency scales of the spectrum. Computations yield $\lambda=0.16$ for the mean JONSWAP spectrum and $\lambda=0.12$ for the Pierson-Moskowitz spectrum.

From (4.5) the momentum input by the wind in the range $\sigma>2.5 \sigma_{m}$ is found to be

$$
\tau_{\mathrm{in}}^{\mathrm{hf}}=0.06 \varrho_{a} \alpha\left(g / \sigma_{m}\right)^{2}\left\{1-0.3 g / \sigma_{m} U\right\} .
$$

The momentum loss due to wave breaking follows similarly from (3.10),

$$
\tau_{\mathrm{ds}}^{\mathrm{hf}}=-0.34 \eta \alpha \varrho_{w} g^{2} / \sigma_{m} .
$$

Thus the equilibrium condition $\tau_{\mathrm{ds}}^{\mathrm{hf}}=-\tau_{\mathrm{nl}}^{\mathrm{hf}}-\tau_{\mathrm{in}}^{\mathrm{hf}}$ yields

$$
\eta=\sigma_{m}^{-1}\left\{2.2 \times 10^{-4}\left(1-0.3 g / \sigma_{m} U\right)+2 \alpha^{2} \lambda\right\} .
$$

Although $\eta$ is determined by the energy balance at high frequencies, the Expression (5.4) depends not only on $\alpha$ but also on the peak frequency $\sigma_{m}$. This arises through the dependence of the nonlinear energy transfer at high frequencies on interactions with wave components in the spectral peak. Thus the equilibrium range of the spectrum cannot be separated dynamically from the low-frequency part of the spectrum.

Equation (5.4) is based on a particular structure of the energy balance which takes account of the principal source functions identified in wave-growth studies at intermediate and larger fetches. Laminar viscous dissipation has been ignored. It is possible that additional processes with less pronounced signatures may also modify these results. For example, no account has been taken of non-conservative WKB-type interactions between waves of widely differing wavelengths (Phillips, 1963; LonguetHiggins, 1963; Hasselmann, 1971).

For a $k^{-4}$ spectrum (3.8a) the principal momentum loss by laminar viscous dissipation lies in the gravity-capillary transition region of the spectrum. Taking an equilibrium constant $\alpha$ in this range of order 0.03 (Phillips, 1966), it is found that the viscous momentum loss can account for all of the momentum transfer across the airsea interface for wind speeds less than about $4 \mathrm{~m} \mathrm{~s}^{-1}$ - which is of approximately the same magnitude as the critical wind speed at which white caps are first observed to appear. However, the value of $\alpha$ is very uncertain in this region of the spectrum.

If the wind input is concentrated in the gravity-wave region of the spectrum, the 
momentum dissipated by viscosity would need to be transferred to the gravity-capillary waves by some form of nonlinear coupling. Conservative third-order interactions between gravity waves alone are unable to bridge the wide spectral gap involved. It is conceivable that second-order interactions between gravity-capillary waves may transfer energy from short gravity waves into the gravity-capillary range (cf., Valenzuela and Laing, 1972). An alternative mechanism is non-conservative WKB interactions, although little is known experimentally about the modulation of very short waves by longer gravity waves. Nevertheless, it may be expected that some fraction of the momentum imparted to the principal wind-sea components by the wind is transferred to very short gravity-capillary waves, where it is dissipated, and that (5.4) should be modified accordingly. The correction cannot be determined with presently available data, but is probably small except for light winds and very small fetches.

\section{The $\sigma^{-5}$ Power Law}

The $\sigma^{-5}$ dependence of the wave spectrum for frequencies beyond the wind-wave peak has been verified in innumerable measurements. It is therefore of interest to investigate whether the power law can be understood on the basis of the energy balance outlined in the previous section. The non-local nature of this balance in the wave-number domain and the observed fetch dependence of $\alpha$ (and also the anisotropy of the directional spectrum) argue against the simple dimensional explanation originally proposed by Phillips (1958). However, it can be shown that the suggested energy balance is consistent with a $\sigma^{-5}$ spectrum in the sense that only this power law yields a balance of both the integrated energy and momentum transfer rates.

The integrated energy and momentum transfer for a given source function $S_{\alpha}$ may be used to define a mean frequency $\bar{\sigma}_{\alpha}$ of the distribution $S_{\alpha}$ through

$$
\frac{\bar{\sigma}_{\alpha}}{g}=\left[\int_{0}^{\infty} \frac{\sigma}{g} \mathrm{~d} \sigma \int_{-\pi}^{\pi} S_{2, \alpha} \cos \theta \mathrm{d} \theta\right] / \int_{0}^{\infty} \mathrm{d} \sigma S_{\alpha} .
$$

Consider now a spectrum of the form



This yields for the wave-breaking source function (3.10) a mean frequency

$$
\bar{\sigma}_{\mathrm{ds}}=\left\{\begin{array}{lll}
\mu \sigma_{m} \frac{n-3}{n-4} & \text { for } & n>4 \\
\gg \sigma_{m} & \text { for } & n<4
\end{array}\right.
$$

where $\mu$ is a factor less than but near to unity which depends on the directional distribution (for a $\cos ^{2} \theta$ spreading factor and $\eta$ independent of $\theta, \mu=0.9$ ). For $n<4$, the momentum integral diverges at high frequencies and $\bar{\sigma}_{\mathrm{ds}}$ depends on the high-frequency cut-off of the spectrum (or the relation (3.10)). 
From the general structure of the energy balance at intermediate and very large fetches, it can be concluded that in both fetch regions, $\bar{\sigma}_{\mathrm{ds}} \approx 2 \sigma_{m}$. In the intermediate fetch range, $\bar{\sigma}_{\mathrm{ds}}$ is about 1.5 times greater than $\bar{\sigma}_{\text {in }}$, which itself is approximately equal to $1.5 \sigma_{m}$, on account of the imbalance $S>0$ at low frequencies. For a fully developed equilibrium spectrum, on the other hand, the integrated energy and momentum input are both balanced by the dissipation, since $S \equiv 0$ and the nonlinear transfer conserves energy and momentum. Hence $\bar{\sigma}_{\mathrm{ds}}=\bar{\sigma}_{\mathrm{in}}$, which is found to be approximately $2 \sigma_{m}$ for an input source function (4.5). Thus in both cases, $\bar{\sigma}_{\mathrm{ds}} \approx 2 \sigma_{m}$, which implies $n \approx 5$ according to (6.1).

Although this value is clearly only approximate, the exponent is reasonably well defined, since $\sigma_{\mathrm{ds}}$ changes rather rapidly with $n$. For $n=4$, Equation (6.1) yields $\bar{\sigma}_{\mathrm{ds}} \gg \sigma_{m}$, and for $n=6, \bar{\sigma}_{\mathrm{ds}}=1.4 \sigma_{m}$. Thus the bounds may perhaps be estimated as $4.5<n<$ $<5.5$, which is of the same order as the observed variation in $n$ (cf., Phillips, 1966).

\section{Conclusions}

Under rather general assumptions, the source function for wave breaking was shown to be of the form $S_{\mathrm{ds}}=-\eta \sigma^{2} E(k)$. A quasi-linear source function of this type is characteristic for any non-expansible interaction process which is weak-in-the-mean. The quadratic frequency dependence of the damping coefficient in the case of wave breaking applies for wavelengths and periods which are large compared with the white-cap scales. The coefficient $\eta$ is a function of the overall wave statistics and is constant, except for a directional dependence, for a given wave state.

The form of the white-capping source function agrees well with the structure of the energy balance derived from JONSWAP and other wave-growth studies, assuming a linear form $S_{\mathrm{in}}=\beta E(k)$ for the input source function. Completion of the energy balance then yields an indirect determination of $\eta$ in terms of the atmospheric input and nonlinear transfer.

If $S_{\text {in }}$ is linear, it can be deduced from the wave-growth data that essentially all of the momentum transferred across the air-sea interface enters the wave field. For fetchlimited and fully-developed spectra in a uniform, stationary wind field, the scales of the spectra vary in such a manner that the resultant drag coefficient remains approximately constant for all fetches and wind speeds. However, for an arbitrary nonuniform, time-dependent wind field, this will generally not be the case. Thus an accurate parameterisation of the air-sea momentum transfer will need to incorporate the wave spectrum as well as the wind speed.

The proposed energy balance, including the wave-breaking source function, was found to be consistent with a $\sigma^{-5}$ equilibrium spectrum, which was explained here not in the Phillips sense as a local balance controlled primarily by wavebreaking, but as a non-local equilibrium involving the atmospheric input and the nonlinear transfer as well as the dissipation by wave breaking.

The corresponding source function set provides a dynamically consistent basis for wave-prediction models. However, several hypotheses were still needed to close the 
energy balance, in particular concerning the form of the input source function. Further air-sea interaction studies, including the direct determination of $S_{\text {in }}$ from cross-correlation measurements of wave height and surface pressure, are needed to resolve these uncertainties.

\section{References}

Barnett, T. P.: 1968, 'On the Generation, Dissipation and Prediction of Ocean Wind Waves', J. Geophys. Res. 73, 513-530.

Barnett, T. P. and Wilkerson, J. C.: 1967, 'On the Generation of Wind Waves as Inferred from Airborne Radar Measurements of Fetch-Limited Spectra', J. Mar. Res. 25, 292-328.

Brocks, K. and Krügermeyer, L.: 1972, in A. L. Gordon (ed.), 'The Hydrodynamic Roughness of the Sea Surface', Studies in Physical Oceanography, Gordon and Breach, New York, 75-92. (Also Report No. 14, Inst. für Radiometeorologie, Hamburg, 1970.)

Darbyshire, J. and Simpson, J. H.: 1967, 'Numerical Prediction of Wave Spectra over the North Atlantic', Deutsche Hydrog. Z. 20, 18.

Davis, R. E.: 1969, 'On the High Reynolds Number Flow over a Wavy Boundary', J. Fluid Mech. 36, 337-346.

Davis, R. F. : 1970, 'On the Turbulent Flow over a Wavy Boundary', J. Fluid Mech. 42, 721-731.

Dobson, F. W.: 1971a, 'Measurements of Atmospheric Pressure on Wind-Generated Sea Waves', J. Fluid Mech. 48, 91-127.

Dobson, F. W.: 1971b, 'The Damping of a Group of Sea Waves', Boundary-Layer Meteorol. 1, 399410.

Dunckel, M., Hasse, L., Krügemeijer, L., Schriever, D., and Wucknitz, J.: 1974, 'Turbulent Fluxes of Momentum, Heat and Water Vapor in the Atmospheric Surface Layer at Sea During ATEX', Boundary-Layer Meteorol., this issue p. 81.

Elliott, J. A.: 1972, 'Microscale Pressure Fluctuations Near Waves Being Generated by the Wind', J. Fluid Mech. 54, 427-448.

Ewing, J. A.: 1971, 'A Numerical Wave Prediction Method for the North Atlantic Ocean', Deutsche Hydrog. Z. 24, 241-261.

Gelci, R., Cazalé, H., and Vassal, J.: 1957, 'Prévision de la Houle. La Méthode des Densités Spectroangulaires', Bull. Inform. Comité Central Oceanogr. d'Étude Cotes 9, 416.

Gelci, R. and Devillaz, E.: 1970, 'Le Calcul Numérique de l'État de la Mer', Houille blanche 25, 117.

Harris, D. L.: 1966, 'The Wave Driven Wind', J. Atmos. Sci. 23, 688 -693.

Hasse, L.: 1970, 'On the Determination of the Vertical Transports of Momentum and Heat in the Atmospheric Boundary Layer at Sea', Tech. Rep. 188, Dept. Oceanogr., Oregon State Univ., Ref. No. 70-20. (Also Hamburger Geophys. Einzelschr. 11 (1970).)

Hasselmann, K.: 1960, 'Grundgleichungen der Seegangsvoraussage. Schiffstechnik 7, 191-195.

Hasselmann, K.: 1962, 'On the Non-Linear Energy Transfer in a Gravity-Wave Spectrum. I: General Theory', J. Fluid Mech. 12, 481-500.

Hasselmann, K.: 1963a, 'On the Non-Linear Energy Transfer in a Gravity-Wave Spectrum. II: Conservation Theorems, Wave-Particle Correspondence, Irreversibility', J. Fluid Mech. 15, 273-281.

Hasselmann, K.: 1963b, 'On the Non-Linear Energy Transfer in a gravity-wave Spectrum. III: Computation of the Energy Flux and Swell-Sea Interaction for a Neumann Spectrum', J. Fluid Mech. 15, 385-398.

Hasselmann, K.: 1967, 'Nonlinear Interactions Treated by the Methods of Theoretical Physics (with Application to the Generation of Waves by Wind)', Proc. Roy. Soc. A 299, 77-100.

Hasselmann, K.: 1968, in M. Holt (ed.), 'Weak-Interaction Theory of ocean Waves', Basic Developments in Fluid Dynamics 2, 117-182.

Hasselmann, K.: 1971, 'On the Mass and Momentum Transfer Between Short Gravity Waves and Larger-Scale Motions', J. Fluid Mech. 50, 189-206.

Hasselmann, K. and Collins, J. I.: 1968, 'Spectral Dissipation of Finite-Depth Gravity Waves due to Turbulent Bottom Friction', J. Mar. Res. 26, 1-12.

Hassemann, K., Barnett, T. P., Bouws, E., Carison, H., Cartwright, D. E., Enke, K., Ewing, J. A., Gienapp, H., Hasselmann, D. E., Kruseman, P., Meerburg, A., Müller, P., Olbers, D. J., Richter, K., Sell, W., and Walden, H.: 1973, 'Measurements of Wind-Wave Growth and Swell Decay During the Joint North Sea Wave Project (JONSWAP)', Deutsche Hydrog. Z., Suppl. $A\left(8^{\circ}\right)$, No. 12. 
Inoue, T.: 1967, 'On the Growth of the Spectrum of a Wind Generated Sea According to a Modified Miles-Phillips Mechanism and its Application to Wave Forecasting', Geophys. Sci. Lab. Rep. No. TR 67-5.

Jeffreys, J.: 1924, 'On the Formation of Waves by Wind' Proc. Roy. Soc. A 107, 189-206.

Lai, R. J. and Shemdin, O. H.: 1971, 'Laboratory Investigation of Air Turbulence over Simple Water Waves', J. Geophys. Res. 76, 7334-7350.

Lamb, H.: 1932, Hydrodynamics, 6th ed., Cambridge University Press.

Long, R.: 1971, 'On Generation of Ocean Waves by a Turbulent Wind', Ph. D. Thesis, Univ. Miami.

Longuet-Higgins, M. S.: 1963, 'The Generation of Capillary Waves by Steep Gravity Waves', J. Fluid Mech. 16, 138-159.

Longuet-Higgins, M. S.: 1969, 'On Wave-Breaking and the Equilibrium Spectrum of Wind-Generated Waves', Proc. Roy. Soc. A 310, 151-159.

Longuet-Higgins, M. S.: 1973a, 'On the Form of the Highest Progressive and Standing Waves in Deep Water', Proc. Roy Soc. A 331, 445-546.

Longuet-Higgins, M. S.: 1973b, 'A Model of Flow Separation at a Free Surface', J. Fluid Mech. 57, 129-148.

Miles, J. W.: 1957, 'On the Generation of Surface Waves by Shear Flow', J. Fluid Mech. 3, 185-204.

Mitsuyasu, H.: 1968, 'On the Growth of the Spectrum of Wind-Generated Waves', I', Rep. Res. Inst. Appl. Mech., Kyushu Univ. 16, 459-482.

Mitsuyasu, H.: 1969, 'On the Growth of the Spectrum of Wind-Generated Waves, II', Rep. Res. Inst. Appl. Mech., Kyushu Univ. 17, 235-248.

Mitsuyasu, H., Nakayama, and Komori, T.: 1971, 'Observations of the Wind and Waves in Hakata Bay', Rep. Res. Inst. Appl. Mech., Kyushu Univ. 19, 37-74.

Peierls, R. E.: 1955, Quantum Theory of Solids, Oxford, Univ. Press.

Phillips, O. M.: 1958, 'The Equilibrium Range in the Spectrum of Wind-Generated Waves', J. Fluid Mech. 4, 426-434.

Phillips, O. M.: 1959, 'The Scattering of Gravity Waves by Turbulence', J. Fluid Mech. 5, 177-192.

Phillips, O. M.: 1963, 'On the Attenuation of Long Gravity Waves by Short Breaking Waves', J. Fluid Mech. 16, 321-332.

Phillips, O. M.: 1966, The Dynamics of the Upper Oceam, Camb. Univ. Press.

Pierson, W. J., Jr. and Moskowitz, L.: 1964, 'A Proposed Spectral Form for Fully Developed Wind Seas Based on the Similarity Theory of S.A. Kitaigorodskii', J, Geophys. Res. 69, 5181-5190.

Pierson, W. J., Jr., Tick, L. J., and Baer, L.: 1966, 'Computer Based Procedure for Preparing Global Wave Forecasts and Wind Field Analysis Capable of Using Wave Data Obtained from a Spacecraft', Sixth Symposium on Naval Hydrodynamics, Washington, D.C., 499.

Pond, S., Phelps, G. T., Paquin, J. E., McBean, G., and Stewart, R. W.: 1971, 'Measurements of the Turbulent Fluxes of Momentum, Moisture, and Sensible Heat over the Ocean', J. Atmos. Sci. 28, 901-917.

Ross, D. B., Cardone, V. J., and Conaway, J. W. Jr.: 1970, 'Laser and Microwave Observations of Sea-Surface Conditions for Fetch-limited 17- to 25-m s' ${ }^{-1}$ Winds', IEEE Trans. GE-8, 326-336.

Schule, J. R., Simpson, L. S., and Deleonibus, P. S. : 1971, 'A Study of Fetch-Limited Wave Spectra with an Airborne Laser', J. Geophys. Res. 76, 4160-4171.

Schwarz, L. W.: 1972, ‘Analytic Continuation of Stokes' Expansion for Gravity Waves', Ph. D. dissertation, Stanford Univ.

Smith, S. D.: 1970, 'Thrust-Anemometer Measurements of Wind Turbulence, Reynolds Stress and Drag Coefficient over Sea', J. Geophys. Res. 75, 6758-6770.

Snyder, R. L.: 1973, 'A Field Study of the Atmospheric Pressure Field Above Surface Waves', Submitted to J. Mar. Res.

Snyder, R. L. and Cox, C. S.: 1966, 'A Field Study of the Wind Generation of Ocean Waves', J. Mar. Res. 24, 141-178.

Townsend, A. A.: 1972, 'Flow in Deep Turbulent Boundary over a Surface Disturbed by Water Waves', J. Fluid Mech. 55, 719-735.

Valenzuela, G. R. and Laing, M. B.: 1972, "Non-Linear Energy Transfer in Gravity-Capillary Wave Spectra, with Applications', J. Fluid Mech. 54, 507-520.

Volkov, Yu. A. and Mordukhovich, M. I.: 1971, 'Spectra of Turbulent Fluxes of Momentum and Heat over a Wavy Sea Surface', Izv. Atmos. Ocean. Phys. 7, 18-17. 